\title{
Pressurised hot water extraction in continuous flow mode for thermolabile compounds: extraction of polyphenols in red onions
}

\author{
Jiayin Liu • Margareta Sandahl • Per J. R. Sjöberg • \\ Charlotta Turner
}

Received: 27 June 2013 /Revised: 11 September 2013 / Accepted: 13 September 2013 /Published online: 4 October 2013

(C) The Author(s) 2013. This article is published with open access at Springerlink.com

\begin{abstract}
Extraction and analysis of labile compounds in complex sample matrices, such as plants, is often a big analytical challenge. In this work, the use of a "green and clean" pressurised hot water extraction (PHWE) approach performed in continuous flow mode is explored. Experimental data for extraction and degradation kinetics of selected compounds were utilised to develop a continuous flow extraction (CFE) method targeting thermolabile polyphenols in red onions, with detection by high-performance liquid chromatography (HPLC)-diode array detection (DAD)-mass spectrometry (MS). Water containing ethanol and formic acid was used as extraction solvent. Method performance was focused on extraction yield with minimal analyte degradation. By adjusting the flow rate of the extraction solvent, degradation effects were minimised, and complete extraction could be achieved within $60 \mathrm{~min}$. The CFE extraction yields of the polyphenols investigated were $80-90 \%$ of the theoretically calculated quantitative yields and were significantly higher than the yields obtained by conventional methanol extraction and static batch extraction (70-79 and 58-67\% of the theoretical yields, respectively). The precision of the developed method was lower than $8 \%$ expressed as relative standard deviation.
\end{abstract}

Published in the topical collection Challenges and New Directions in Analytical Sample Preparation with guest editors Astrid Gjelstad and Stig Pedersen-Bjergaard.

Electronic supplementary material The online version of this article (doi:10.1007/s00216-013-7370-7) contains supplementary material, which is available to authorized users.

J. Liu $\cdot$ M. Sandahl $\cdot$ C. Turner $(\triangle)$

Department of Chemistry, Centre for Analysis and Synthesis, Lund

University, P.O. Box 124, 22100 Lund, Sweden

e-mail: charlotta.turner@chem.lu.se

\section{P. J. R. Sjöberg}

Department of Chemistry - BMC, Analytical Chemistry, Uppsala

University, P.O. Box 599, 75124 Uppsala, Sweden
Keywords Anthocyanins · Quercetin · Polyphenols · Extraction $\cdot$ Degradation $\cdot$ Kinetics

\section{Introduction}

Polyphenols are a group of high-value compounds that can be extracted from by-products originating from the food and agricultural industry. Conventionally, they are extracted by an organic solvent, such as methanol, at low temperature over a long period of time. Pressurised hot water extraction (PHWE) is an attractive and environmentally sustainable alternative for the extraction of these compounds. At elevated temperature, the viscosity and surface tension of water decrease, while diffusivity and solubility of the phenolic compounds increase, providing faster and more complete extraction.

PHWE of polyphenols has been studied in numerous literatures, among which intensive method optimisation could also be found. One important issue of PHWE is that many of the phenolic compounds are thermolabile, thus limiting to a certain extent the use of ordinary batch-format (static) PHWE. Optimising extraction of these thermolabile compounds is dubious if degradation is not considered. By utilising previous knowledge from the literature that degradation of polyphenols follows first-order kinetics, there is a potential to further improve the extraction yield. Attempts have been made to mathematically calculate the theoretical yield of thermolabile polyphenols by integrating degradation kinetics with extraction data [1], but the potential to improve the extraction yield has yet to be verified.

In this study, an extraction method with maximum polyphenol yield and minimum degradation is presented utilising continuous flow PHWE. Minimising the degradation of sensitive compounds during extraction was explored by constructing and evaluating the performance of a continuous flow extraction (CFE) system. Target polyphenol compounds in red onions 
include anthocyanins and flavonols. With the CFE system, it is possible to easily control the residence time of the polyphenols in the system by varying the flow rate of the solvent, thus providing the means necessary to minimise the degradation of these thermolabile compounds. Extraction yields were compared to those theoretically calculated using a method presented previously, which combined extraction and degradation kinetics [1], and also to those yields obtained by a conventional solvent extraction method.

The aim of this study was to develop an accurate PHWE method suitable for quantification of polyphenols in red onion and also applicable to the most thermolabile compounds.

\section{Materials and methods}

\section{Chemicals}

Anthocyanin standard cyanidin-3-O-glucoside chloride (C3G) of high-performance liquid chromatography (HPLC) grade was purchased from Extrasynthese (Genay, France). Quercetin dihydrate (Q) was purchased from Sigma-Aldrich (Steinheim, Germany). Quercetin-4'-glucoside (QMG) and quercetin-3,4'-diglucoside (QDG) were purchased from Polyphenols Laboratories AB (Sandnes, Norway). Acetonitrile $(99.8 \%)$ and formic acid (98 \%) were purchased from Merck (Darmstadt, Germany). Ethanol (99.7 vol\%) was obtained from Solveco AB, Sweden. All solvents used were of HPLC grade. Ultrapure water was obtained from a purification system (Milli-Q; Millipore, Bedford, MA). Nitrogen (99.996 vol\%) was from AGA (Stockholm, Sweden).

\section{Sample pretreatment}

Red onions (Allium cepa L.) were purchased from a local supermarket in Lund, Sweden. A batch of fresh red onions was cut into small pieces of average size $(5 \mathrm{~mm} \times 5 \mathrm{~mm} \times$ $2 \mathrm{~mm}$ ) and stored at $-18{ }^{\circ} \mathrm{C}$ until usage.

Continuous flow extraction of polyphenols from red onion

In the CFE system (shown in Electronic Supplementary Material Fig. S1A), the extraction solvent (water, ethanol and formic acid (94:5:1, $v / v / v)$ ), kept in an airtight solvent bottle, was preheated with the aid of a heating plate to $75^{\circ} \mathrm{C}$ before it entered the oven, which was held at $110^{\circ} \mathrm{C}$, containing the preheating coil and extraction cell. Addition of ethanol in the extraction solvent will increase the solubility of flavonol compounds, as the solubilities of these substances are much lower in pure water. Adding formic acid also increases the solubility and/or stability of the flavonols [2].

Onion samples of $2.0 \mathrm{~g}$ were put into the stainless steel extraction cell. To avoid clogging in the tubes by onion pieces carried away by the extraction solvent, the extraction cell was sealed by micropore filters ( $1 \mathrm{~mm}$ thick, $12 \mathrm{~mm}$ in diameter) and screw caps at both ends.

Flow rates investigated were 2,3 and $4 \mathrm{~mL} \mathrm{~min}^{-1}$. As soon as the oven temperature was switched on, the LC pump was started to continuously flush out extracted compounds. It took about $3 \mathrm{~min}$ for the extraction solvent to reach $110^{\circ} \mathrm{C}$ in the GC oven. Timing started when the first drop of extract appeared at the end of the collection tube. Samples were collected in $50-\mathrm{mL}$ conical tubes wrapped in aluminium foil every $6 \mathrm{~min}$ up to $60 \mathrm{~min}$. From each tube, $1 \mathrm{~mL}$ of sample was taken out for chromatographic analysis. All experiments in this study were performed in triplicates.

Static batch extraction experiments

Batch extraction experiments were performed in a highpressure batch reactor (see Electronic Supplementary Material Fig. S1B) with a cell volume of $100 \mathrm{~mL}$ (Mini Reactor, Autoclave Engineers, Erie, PA, USA). Onion samples of $2.0 \mathrm{~g}$ were extracted by an $80-\mathrm{mL}$ solution of water, ethanol and formic acid $(94: 5: 1, v / v / v)$ at $110{ }^{\circ} \mathrm{C}$ for $120 \mathrm{~min}$. Detailed experimental procedure and the derivations of theoretical total extraction curves can be found in our previous study [1].

Degradation experiments of polyphenol standards in solution

Individual standards of QDG and C3G were dissolved in a mixture of water, ethanol and formic acid (94:5:1, v/v/v) to a concentration of about $25 \mu \mathrm{M}$. First, $80 \mathrm{~mL}$ of standard solution was put into the reactor (Electronic Supplementary Material Fig. S1B) and heated to $110{ }^{\circ} \mathrm{C}$, and ten sample fractions were collected during $100 \mathrm{~min}$ (i.e., every tenth minute). Collected samples were then analysed by HPLCdiode array detector (DAD). The experiment was performed for the two standards, individually.

Conventional solvent extraction of polyphenols from red onions

Conventional extraction was carried out using a method adapted from Spigno et al. [3]. An extraction solvent of $100 \mathrm{~mL}$ of methanol (1\% formic acid) and onion samples of $2.5 \mathrm{~g}$ were used to keep the liquid/solid ratio the same as in the extraction experiment in section "Static batch extraction experiments". The extraction was performed under protection from light at $4{ }^{\circ} \mathrm{C}$ in the fridge for $72 \mathrm{~h}$ to achieve sufficient extraction. Occasional shaking was applied from time to time. After $72 \mathrm{~h}$ the extracts were centrifuged, and $1 \mathrm{~mL}$ of the supernatant was analysed by HPLC-DAD. 


\section{HPLC-DAD-MS analysis}

Chromatographic analysis was performed on a Dionex Ultimate 3000 HPLC equipped with quaternary pump, online degasser, autosampler, DAD and Chromeleon Chromatography Data System (Dionex Softron GmbH, Germering, Germany). Separation of the sample extracts was conducted on a Synergi Max reversed-phase HPLC column $(2 \mathrm{~mm} \times 100 \mathrm{~mm}$, $2.5 \mu \mathrm{m}$, Phenomenex, Torrance, CA, USA). Elution was performed using mobile phase A ( $5 \%$ formic acid in $\mathrm{H}_{2} \mathrm{O}$, in volume percent) and mobile phase $\mathrm{B}$ ( $5 \%$ formic acid in $\mathrm{MeOH}$, in volume percent). The optimal elution profile was a multistep linear solvent gradient: $0-5 \mathrm{~min}, 0 \% \mathrm{~B} ; 5-15 \mathrm{~min}$, 0-5 \% B; 15-25 min, 5-10\% B; 25-45 min, 10-20\% B; 45$55 \mathrm{~min}, 20-25 \% \mathrm{~B}$; 55-85 min, 25-95\% B; 85-90 min, $95 \%$ B; 90-91 min, 95-0 \% B and 91-120 min, $0 \%$ B. The flow rate was $200 \mu \mathrm{L} / \mathrm{min}$, and total DAD spectra were monitored in $3 \mathrm{D}$ mode, ranging from 200 to $680 \mathrm{~nm}$. The main wavelengths monitored were $290 \mathrm{~nm}$ for phenolics, $350 \mathrm{~nm}$ for flavonols and $520 \mathrm{~nm}$ for anthocyanins.

The mass spectrometer was a 3200 Linear Ion Trap Quadrupole Mass Spectrometer (3200 QTRAP, Applied Biosystems/ MDS Sciex Instruments, Concord, ON, Canada). A TurboV electrospray source was used in positive mode to produce ions, which then entered the QTRAP for information-dependent acquisition based on enhanced mass spectrometry (EMS) as survey scan followed by enhanced product ion (EPI) scan. The mass spectrometry (MS) parameters were applied as follows: source temp $=600{ }^{\circ} \mathrm{C}$, curtain gas $=15 \mathrm{psi}$, ion source gas $1=60 \mathrm{psi}$, ion source gas $2=60 \mathrm{psi}$, ion spray voltage $=5,500 \mathrm{~V}$, collision gas $=$ high, declustering potential $=30 \mathrm{~V}$, entrance potential $=10 \mathrm{~V}$ and collision energy $=30 \mathrm{~V}$. The analysis was conducted in negative mode as well, and the parameters applied in negative mode were source temp $=600{ }^{\circ} \mathrm{C}$, curtain gas $=15 \mathrm{psi}$, ion source gas $1=$ $60 \mathrm{psi}$, ion source gas $2=60 \mathrm{psi}$, ion spray voltage $=-4,500 \mathrm{~V}$, collision gas $=$ high, declustering potential $=-30 \mathrm{~V}$, entrance potential $=-10 \mathrm{~V}$, and collision energy $=-30 \mathrm{~V}$.

\section{Compound quantification}

Quantification of anthocyanins was performed by HPLC-DAD using a calibration curve of $\mathrm{C} 3 \mathrm{G}$ in the range of $1-50 \mu \mathrm{mol} \mathrm{L}^{-1}$, and yields were expressed as milligrams of $\mathrm{C} 3 \mathrm{G}$ equivalents per gram of fresh weight red onion. Quantification of flavonols QDG, QMG and Q was performed by calibration curves of corresponding standards in the same range as above, and yields were expressed as milligrams per gram of fresh weight red onion.

\section{Results and discussion}

A typical chromatographic profile of red onion extract is shown in Electronic Supplementary Material Fig. S2. With the aid of
DAD, selective detection could be achieved for anthocyanins and flavonols by obtaining chromatograms at suitable wavelengths. Identification of the selected peaks was based on comparison of retention times with chemical standards and/or MS/MS data. For example, an MS spectrum of QDG is shown in Electronic Supplementary Material Fig. S3. This demonstrated a deprotonated molecule of $\mathrm{m} / \mathrm{z} 625$ and two significant fragment ions of $\mathrm{m} / \mathrm{z} 463$ and 301 in negative mode, which indicated the cleavage of glycosidic bonds that occurred between the quercetin backbone and the glucose moiety at both 3 and $4^{\prime}$ positions.

Anthocyanins and flavonoids are the main components extracted from red onion, as was shown in Electronic Supplementary Material Table S1. Among the seven identified anthocyanins, four major ones, namely $\mathrm{C} 3 \mathrm{G}$, cyanidin-3laminaribioside $(\mathrm{C} 3 \mathrm{~L})$, cyanidin-3-(6"-malonoylglucoside) (C3MG) and cyanidin-3-(6"-malonoyl-laminaribioside) (C3ML), were chosen to calculate the theoretical yield. QDG, QMG and Q are all present in large quantity in red onion, but $\mathrm{QMG}$ and $\mathrm{Q}$ can also be formed through degradation of QDG, which makes the calculation of theoretical extraction curve unfeasible, since the formation rate is unknown. Therefore, the theoretical extraction yield was only calculated for QDG (see Supplementary Material).

To optimise the CFE method, the flow rate of the solvent was altered to achieve the best polyphenol yield. The CFE temperature was set to $110^{\circ} \mathrm{C}$ because studies had shown that at higher temperature $\left(>120^{\circ} \mathrm{C}\right)$, the degradation of polyphenols was severe, and at lower temperature $\left(<80^{\circ} \mathrm{C}\right)$, the extraction was not fast enough [2]. Investigation of individual anthocyanins and flavonols rather than the total polyphenol amount enabled the quantitative determination of the single species, which is important since the degradation rates of different compounds differ distinctively. A graph of the extraction yield of $\mathrm{C} 3 \mathrm{G}$ as a function of time and extraction volume is shown in Fig. 1. As can be seen in Fig. 1a, increasing the flow rate will accelerate the mass transfer and decrease the residence time of $\mathrm{C} 3 \mathrm{G}$ in the $\mathrm{CFE}$ system, thus reducing the degradation and increasing the extraction yield. The residence time is defined as the time it takes for individual components to elute from the extraction system, here calculated by taking the length of the extraction vessel divided by the linear flow rate. Since the degradation rate varies for different compounds, the maximum allowable residence time to achieve a certain yield can also be different. In Fig. 1b, the $\mathrm{C} 3 \mathrm{G}$ yield per extraction volume was independent of the flow rate up to $36 \mathrm{~mL}$, which indicates an efficient removal of the target compounds loosely bound to the sample matrix, and the extraction rate is controlled primarily by a "solubility/elution" step [4]. At higher flow rates, the removal of extracted compound out of the matrix is faster because of a higher concentration gradient. In addition, the residence time in the extraction cell is shorter at higher flow rate, therefore minimising the 

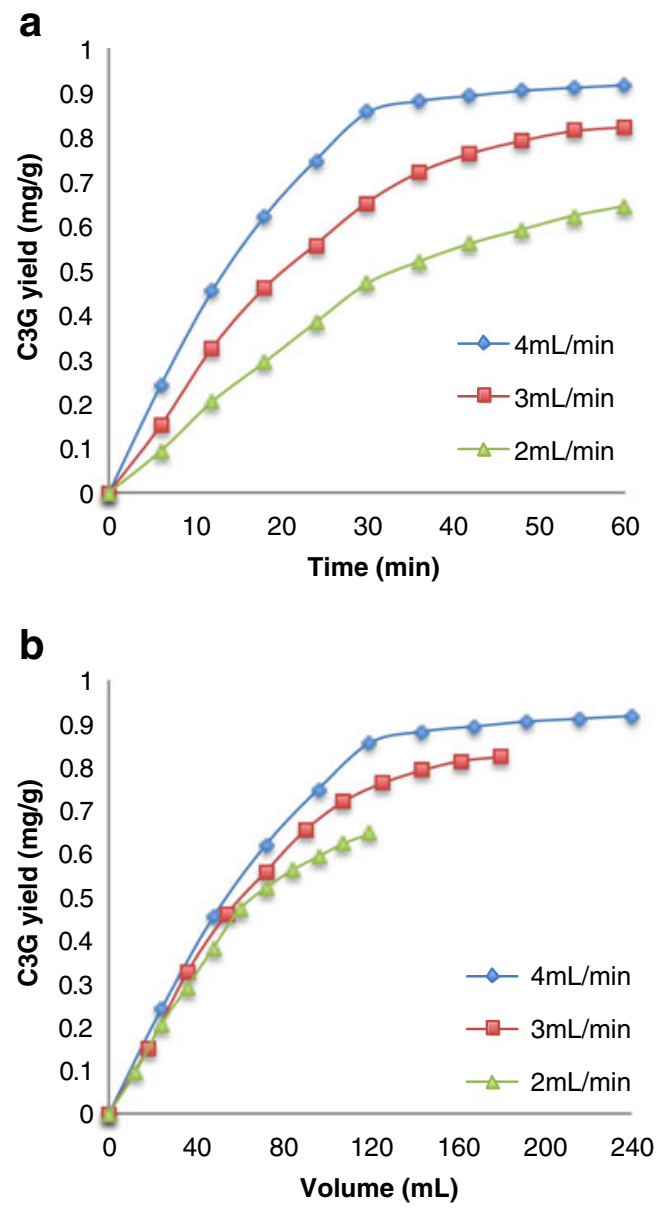

Fig. 1 Extraction yield of $\mathrm{C} 3 \mathrm{G}$ as a function of time (a) and solvent volume (b) at different flow rates at $110{ }^{\circ} \mathrm{C}$

effect of the degradation. However, from 42 to 60 min using a flow rate of $4 \mathrm{~mL} \mathrm{~min}^{-1}$ for example (Fig. 1a), the extraction yield did not increase more than $7 \%$. It means that this fraction of $\mathrm{C} 3 \mathrm{G}$ is more tightly attached to the onion matrix, and removal of $\mathrm{C} 3 \mathrm{G}$ from the system requires desorption/ diffusion of the compound from the matrix, which limits the extraction rate [4]. Since the extraction only increases by $7 \%$ from 42 to $60 \mathrm{~min}$, an extraction time longer than $60 \mathrm{~min}$ was expected to be less significant. Increasing the flow rate at the desorption/diffusion-controlled step will not increase the extraction rate, but faster transportation of the polyphenols at higher flow rate will still help to prevent the degradation (see Fig. 1b).

A strategy to optimise the extraction method is (1) to theoretically calculate the possible quantitative yield as if there was no degradation and (2) to use the degradation rate of the individual compounds to calculate the maximum allowable residence time to obtain $90 \%$ of the theoretical yield. Theoretical calculation of quantitative yields was performed by utilising the fact that degradation of polyphenols follows first-order kinetics combined with extraction and degradation kinetics in a stirred batch reactor [1]. The theoretical yields of
C3G, C3L, C3MG, C3ML and QDG were calculated using data obtained from the static batch extraction experiment (section "Static batch extraction experiments") and using a mathematical method developed previously (see Supplementary Material Tables S2 and S3) [1]. Degradation rates during extraction of onion samples $\left(k_{\text {ext }}\right)$ were calculated as firstorder kinetics by the last three points of the extraction experiment (section "Static batch extraction experiments"), assuming a complete extraction in the end.

The maximum residence time to obtain $90 \%$ recovery from theoretical yield was calculated to be 36 min for $\mathrm{C} 3 \mathrm{G}$ and $75 \mathrm{~min}$ for QDG using their respective degradation rates $\left(k_{\text {ext }}=0.011 \mathrm{~min}^{-1}\right.$ for $\mathrm{C} 3 \mathrm{G}$ and $0.005 \mathrm{~min}^{-1}$ for QDG), which means that to achieve $90 \%$ recovery, the extraction must be complete before 36 and $75 \mathrm{~min}$ for $\mathrm{C} 3 \mathrm{G}$ and QDG, respectively. This implies that the flow rate must be $4 \mathrm{~mL} \mathrm{~min}^{-1}$ or higher as can be seen in Fig. 1a. In this study, a higher flow rate than $4 \mathrm{~mL} \mathrm{~min}^{-1}$ was not preferred to avoid exhaustion of the pump or further dilution of the extract. QDG has a lower degradation rate compared to all the anthocyanins. Therefore, the extraction solvent flow rate could be lower if only flavonols are the target compounds, which would then have the benefit of less dilution of the extract.

Degradation rates using standard solution $\left(k_{\mathrm{std}}\right)$ of $\mathrm{C} 3 \mathrm{G}$ $\left(k_{\text {std }}=0.019 \mathrm{~min}^{-1}\right)$ and QDG $\left(k_{\mathrm{std}}=0.007 \mathrm{~min}^{-1}\right)$ were also calculated as first-order kinetics by data from the degradation experiment (section "Degradation experiments of polyphenol standards in solution") to investigate the effect of the onion matrix on the degradation kinetics. From the discussion above, it can be concluded that degradation is significantly slower during onion extraction compared to that in standard solution. This indicates that the onion sample matrix has a certain protection effect on the degradation of the polyphenols. The same protective effect of the sample matrix was also observed for $\beta$-carotene, in which the stability decreased when tissues were removed from mango purée [5].

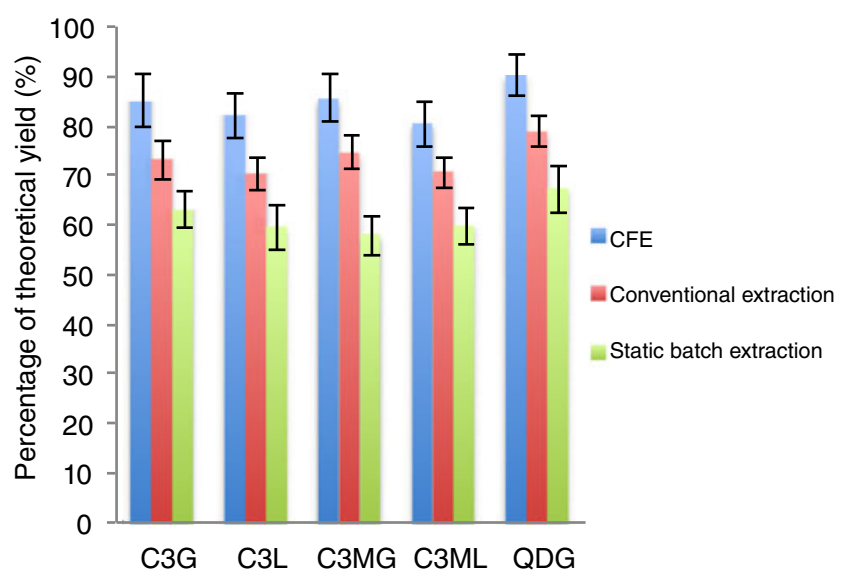

Fig. 2 Polyphenol yields obtained by different extraction methods, expressed as percentage of theoretical yield. Error bars represent standard deviation 
Conventional methanol extraction was also performed to compare with $\mathrm{CFE}$ and static batch extraction. It can be seen in Fig. 2 that $80-90 \%$ of the theoretical yield was obtained by the CFE method for all the polyphenols investigated, while conventional extraction achieved 70-79\% and static batch extraction even lower (58-67\%). Two-sample $t$ tests were applied to the yields obtained by the different methods, and the differences between all methods were statistically significant within $95 \%$ confidence interval. It is noteworthy that after $72 \mathrm{~h}$ of conventional extraction, the onion sample was still lightly coloured which implies insufficient extraction, probably due to strongly bound compounds that could not be removed by leaching, while CFE greatly reduced the extraction time to $60 \mathrm{~min}$ yet still achieving higher extraction yield. The CFE method gives higher yields compared to the conventional solvent extraction method, even though a shorter extraction time is needed. However, one drawback with the CFE system is that higher dilution of the extract occurs compared to a static batch extraction.

\section{Conclusions}

The advantage of the continuous flow system is that the extraction is accomplished in a dynamic flow. Fresh solvent during the CFE process enhances the mass transfer of the target compounds, and degradation is minimised to achieve higher extraction yield compared to static batch PHWE and conventional methanol extraction in a much shorter extraction time. Depending on the degradation rate of the target compounds, the flow rate of the extraction solvent was adjusted accordingly to influence the residence time. Furthermore, the sample matrix proved to have a protective effect on the thermolabile compounds. By comparing CFE, static batch PHWE and conventional methanol extraction, this study provides a new approach in the method development of optimising extraction of polyphenols from complex samples, using "green and clean" pressurised hot water extraction.

Acknowledgments The authors would like to thank Rolf Danielsson for assistance with the theoretical calculations. The Swedish Research Council FORMAS (229-2009-1527), the Swedish Research Council (VR, 2010-5439; 2010-333) and the Swedish Foundation for International Cooperation in Research and Higher Education (STINT, YR20097015) are acknowledged for financial support of the project.

Open Access This article is distributed under the terms of the Creative Commons Attribution License which permits any use, distribution, and reproduction in any medium, provided the original author(s) and the source are credited.

\section{References}

1. Petersson EV, Liu J, Sjoberg PJR, Danielsson R, Turner C (2010) Anal Chim Acta 663:27-32

2. Arapitsas P, Turner C (2008) Talanta 74:1218-1223

3. Spigno G, Tramelli L, De Faveri DM (2007) J Food Eng 81:200-208

4. Hawthorne SB, Galy AB, Schmitt VO, Miller DJ (1995) Anal Chem 67:2723-2732

5. Vásquez-Caicedo AL, Schilling S, Carle R, Neidhart S (2007) Food Chem 102:1172-1186 